\title{
Percepción de igualdad de género en la formación de universitarios del área de la salud
}

\author{
Perception of gender equality in the training of university \\ students in the health area
}

DR. VÍCTOR ALEXANDER QUINTANA LÓPEZ

Universidad Autónoma de Baja California, California , México (vquintana@uabc.edu.mx) (https://orcid.org/0000-0002-5778-1093)

DRA. CECILIA IVONNE BOJÓRQUEZ DÍAZ

Instituto Tecnológico de Sonora, Sonora , México (cecilia.bojorquez@itson.edu.mx) (https:// orcid.org/0000-0003-0237-5079)

DRA, KARINA DE JESÚS DÍAZ LÓPEZ

Universidad Autónoma de Baja California, California, México (diaz.karina52@uabc.edu.mx) (https://orcid.org/0000-0001-6045-2242)

DRA. MARÍA ESTHER MEJÍA LEÓN

Universidad Autónoma de Baja California, California, México (esther.mejia86@uabc.edu.mx) (https://orcid.org/0000-0003-3387-4858)

\section{RESUMEN}

Incluir la perspectiva de género en el currículo educativo de universidades de México es una prioridad apremiante para una sociedad con hombres y mujeres con iguales oportunidades. El objetivo fue analizar la percepción en estudiantes universitarios del área de la salud respecto a perspectiva de género en sus programas educativos. Se realizó un estudio descriptivo-transversal utilizando la escala de Evaluación Sensible a la Formación en Igualdad de Género (ESFIG) en 99 (70,8\% mujeres y 29,2\% hombres) estudiantes de medicina, nutrición, odontología y posgrado en el área de la salud. El 93,4\% mencionó no haber cursado una asignatura sobre género o igualdad de género durante su formación universitaria. En una escala del uno al diez los estudiantes otorgan una calificación de 9,4 a la importancia de la formación para la igualdad de género. Se obtuvo una media de 4,35 del máximo a obtener que era 6 respecto a la percepción que tienen sobre la sensibilidad institucional a la aplicación de la política de igualdad de género en su facultad de adscripción. Al comparar entre los distintos programas educativos respecto a la subescala de sensibilidad institucional a la aplicación de la política de igualdad de género en los centros. Los estudiantes de odontología, a diferencia de los de medicina, perciben que su programa educativo promueve la aplicación de la política de igualdad de género en su currículo ( $p=0.03$ ). Es importante promover 
la aplicación de igualdad de género en los programas educativos de los centros educativos para disminuir las desigualdades entre hombres y mujeres.

\begin{abstract}
Including the gender perspective in the educational curriculum of Mexican universities is an urgent priority for a society with men and women with equal opportunities. The objective was to analyze the perception of university students of health area regarding the gender perspective in their educational programs. A descriptive-cross-sectional study was carried out using the Sensitive Evaluation of Training in Gender Equality (ESFIG) scale in 99 (70.8\% women and 29.2\% men) medicine, nutrition, dentistry, and postgraduate in health area. $93.4 \%$ mentioned not having taken a subject on gender or gender equality during their university education. On a scale of one to ten, the students give a rating of 9.4 to the importance of training for gender equality. An average of 4.35 was obtained from the maximum to obtain, which was 6 regarding the perception they have about the institutional sensitivity to the application of the gender equality policy in their faculty of affiliation. When comparing between the different educational programs regarding the subscale of institutional sensitivity to the application of the gender equality policy in the centers, dentistry students, unlike medicine perceive that their educational program promotes the application of gender equality policy in their curriculum $(p=0.03)$. It is important to promote the application of gender equality in the educational programs of educational centers to reduce inequalities between men and women.
\end{abstract}

\title{
PALABRAS CLAVE/KEYWORDS
}

perspectiva género, estereotipos, diversidad, equidad, roles género, transversalidad./gender perspective, stereotypes, diversity, equity, gender roles, transversality.

\section{INTRODUCCIÓN}

Cuando se hace referencia a la perspectiva de género, inmediatamente salta a la luz el tema de las desigualdades, estas tienen que ver respecto a los derechos y obligaciones que culturalmente se han asignado a hombres y mujeres. Las desigualdades se pueden ver en el hogar, en el ambiente laboral, social, político y en la economía (Ingaramaro, 2013). A pesar que en México se ha trabajado de forma intensa durante las últimas décadas en el tema de perspectiva de género, la realidad es que aún persisten los estereotipos, roles y prejuicios que mantienen en desventaja a las mujeres. Lo anterior impacta de manera negativa en las posibilidades de desarrollo en todos los ámbitos de las personas del sexo femenino (Rodríguez, et al., 2016). 
Nadie pone en tela de juicio que en México se ha avanzado de manera significativa en la búsqueda de que hombres y mujeres tengan las mismas condiciones de igualdad. Sin embargo, sigue predominando una cultura machista discriminatoria que pone en desventaja, inequidad y exclusión a las mujeres. Se requiere que todos los actores responsables de promover y lograr la igualdad de género en el país se comprometan con lograr verdaderos cambios para tener un desarrollo social más completo (Moctezuma-Navarro, et al., 2014).

\section{ANTECEDENTES}

Los centros educativos suelen ser ámbitos de actuaciones en los cuales se busque que haya un equilibrio entre hombres y mujeres, respecto a los roles que hombres y mujeres deben desempeñar en la sociedad. Es en estos centros en los que se deben implementar medidas y actuaciones que lleven a una verdadera igualdad de oportunidades entre hombres y mujeres (Perales, 2012). Desde los años setenta se ha hecho un esfuerzo importante para que la perspectiva de género sea tomada en cuenta en el contexto educativo de México. El camino ha sido complicado, pero por primera vez se incluyó la perspectiva de género en el Plan Nacional de Desarrollo 2013-2018 y en el Programa Sectorial de Educación Pública 20132018. Las personas que conforman las comunidades académicas, han trabajado arduamente por la preocupación que les causa la inequidad de género en el currículo de los programas educativos de las universidades (Camarena-Adame \& Saavedra-García, 2018).

En las universidades de México se requiere que todos los actores reconozcan las desigualdades que existen entre hombres y mujeres en los organigramas, y en general, en la participación de todas las actividades importantes del centro educativo. A medida que ha avanzado el tiempo, en más centros educativos del país se han establecido unidades de género para fomentar actividades encaminadas a lograr la igualdad entre hombres y mujeres, así como para ayudar a resolver situaciones relacionadas con la perspectiva de género. Sin embargo, las actividades de estas unidades muchas veces no se realizan de la forma esperada porque tienen serias restricciones por parte de algunas autoridades. Se requiere para poder lograr una igualdad de género que las universidades reconozcan en qué áreas de las universidades están en desventaja las mujeres y tomar medidas pertinentes para lograr así un desempeño equitativo entre los dos sexos (Cerna, et al., 2017).

Incluir la perspectiva de género en los programas educativos de las universidades no es una tarea sencilla. Requiere en primera instancia que los centros educativos reconozcan que algunos contenidos temáticos del currículo universitario posicionan a las mujeres en una posición de subordinación y desventaja. Lo anterior por la cultura patriarcal que aún predomina en la sociedad y que de alguna manera rige el sistema educativo de muchos países (Jiménez, 2015). Lograr la igualdad entre ambos sexos y que los valores de las mujeres sean respetados por los hombres, no es una tarea exclusiva de las universidades. Se requiere que desde la educación básica los docentes asuman un papel activo en la formación de los estudiantes. Son muchas las causas de las problemáticas de género y son exclusivas de un solo nivel económico o social. Los docentes tienen un papel importante en la educación de género porque ellos pueden lograr en las aulas de clases un cambio cultural positivo que permita sociedades más equitativas (Jiménez-Quenguan, et al., 2020). 
Las universidades de la mayoría de los países tienen actualmente un compromiso importante en la educación con perspectiva de género, sin embargo, pese al gran esfuerzo que se ha hecho, faltan muchos aspectos importantes de lograr. Probablemente se requiera que las universidades se transformen implementando un modelo pedagógico diferente que permita realmente destruir las desigualdades entre mujeres y hombres. Las universidades necesitan cambiar las formas de ser, de pensar y de actuar de todos los actores de las instituciones de educación superior, ello se logra eliminando el currículo oculto de género (Durán, 2022). En un estudio realizado en el Estado de Hidalgo en México se encontró que desde el año 2010 hay un porcentaje mayor de mujeres matriculadas en la universidad, sin embargo, este porcentaje sigue siendo inferior con el de los hombres. Otro de los hallazgos es que las mujeres siguen inscritas en programas educativos orientados al cuidado, educación y bienestar de otros, carreras que son consideradas típicamente femeninas (Castillo \& Reyes, 2022).

En el área de la salud, cada vez hay más mujeres que estudian la carrera de medicina y que ejercen su profesión en el ámbito laboral. Se ha visto que las mujeres que desempeñan esta profesión están más satisfechas con el desarrollo personal que logran a diferencia de los hombres, sin embargo, se encuentran insatisfechas por la falta de igualdad de oportunidades para ocupar puestos de mayor responsabilidad y de toma de decisiones. Dichos puestos siguen reservados para hombres, mientras que a ellas se les ubica en roles de cuidadoras (González-Baltazar, et al., 2015). Actualmente cada vez más estudiantes y docentes se interesan por los temas relacionados con las cuestiones de género. Esto debe ser una pauta que las instituciones educativas aprovechen para capacitar a estudiantes y docentes en estos temas y lograr así una transformación en la sociedad para que sea más tolerante e igualitaria (Canosa-Domínguez, et al., 2020).

Algunas Instituciones de Educación Superior consideran que tener un comité de perspectiva de género o estudiantes y docentes haciendo investigaciones relacionadas con perspectivas de género les permite erradicar las desigualdades entre hombres y mujeres. Se logra una verdadera igualdad de género cuando en la vida cotidiana existe en las aulas de clases una tendencia al equilibrio, tolerancia y respeto entre ambos sexos. Disminuir la discriminación a la mujer no es una tarea sencilla, las sociedades actuales siguen siendo predominantemente patriarcales, por lo que se requiere un trabajo de fondo sobre las estructuras y relaciones jerárquicas en las que la mujer pueda tener las mismas oportunidades que un hombre (Aragón-Macías, et al., 2020).

\section{OBJETIVO}

Analizar la percepción que universitarios del área de la salud tienen respecto a la igualdad de género durante la formación académica. 


\section{METODOLOGÍA}

El diseño de la investigación es no experimental, con enfoque cuantitativo y de tipo descriptivo - transversal. Participaron en total 99 estudiantes del área de la salud, de facultades de medicina y odontología de una universidad pública del norte de México de los siguientes programas educativos: nutrición, odontología y medicina. La media de edad de los participantes fue de 21.5 años. Los estudiantes universitarios fueron invitados a participar por los autores de este artículo.

\section{INSTRUMENTO}

Se utilizó la escala de Evaluación Sensible a la Formación en Igualdad del Género (ESFIG) (Miralles-Cardona, 2020). La ESFIG cuenta con 16 reactivos que se clasifican en tres subescalas con cinco, siete y cuatro reactivos, que miden sensibilidad institucional a la aplicación de la política de igualdad de género en los centros, incorporación de la perspectiva de género en los planes de estudio y la consciencia de desigualdades vinculadas al género en los procesos instructivos. La puntuación mínima a obtener en cada reactivo es de 1 y la máxima de 6.

Procedimiento: Durante dos meses los autores de este artículo invitaron a estudiantes universitarios a responder el instrumento, mismo que fue cargado en un enlace de Google Forms. Para poder responder el instrumento, se solicitó a cada uno de los estudiantes dar su consentimiento informado.

Se realizó estadística descriptiva de los datos para determinar media y desviaciones estándar para cada ítem evaluado y pruebas de t para muestras independientes para comparar las posibles diferencias para cada subescala en relación al sexo de los participantes. Adicionalmente, se llevaron a cabo análisis de varianza (ANOVA) y pruebas de comparación de medias de Tukey para determinar diferencias de percepción de acuerdo a la licenciatura de origen de los participantes. Se consideraron valores de p menores de 0.05 como significativos. Se utilizó el paquete estadístico STATA versión 12.

\section{RESULTADOS}

Las características de los participantes se observan en la Tabla 1. El 70,8\% de los participantes fueron mujeres ( $n=70)$, mientras que 29 participantes fueron hombres, lo que representó un 29\%. En cuanto a la edad promedio, se encontró que fue de 21,5 \pm 4,12. Los programas de estudios evaluados fueron: 38,4\% licenciatura en nutrición, 19,1\% medicina, $33,4 \%$ odontología y 9,1\% posgrado en área de la salud. Respecto a la ocupación, se encontró que el $67,6 \%$ (n=67) solo se dedicaban a estudiar y 32 participantes estudiaban y trabajaban. Cuando se les preguntó sobre si su plan de estudios requería cursar de forma obligatoria alguna asignatura relacionada con los estudios de género se encontró que solo el 13\% 
mencionó que sí mientras que el $86 \%$ no era obligatorio cursar alguna asignatura relacionada con estudios de género. Respecto a si en su plan de estudios había alguna materia optativa sobre género/igualdad de género, se encontró que solo $20 \%$ mencionaron que sí y $79 \%$ que no. Así mismo, se les cuestionó sobre si en algún momento habían cursado alguna asignatura sobre genero/igualdad de género y se observó que solo 6,06\% participantes mencionaron que sí y 93,9\% que no había cursado ninguna asignatura sobre ello. Finalmente, al preguntar sobre la importancia que le otorgaba a la formación para igualdad de género en una escala del 1 al 10, donde 1 se consideró como mínimo y 10 como máximo, el promedio fue de 9,4 \pm 1,31 .

\begin{tabular}{|c|c|}
\hline Caracteristicas & $(\mathbf{n}=99)$ \\
\hline \multicolumn{2}{|l|}{ Sexo $(\%, n)$} \\
\hline Mujer & $70,8 \%(70)$ \\
\hline Hombre & $29,2 \%(29)$ \\
\hline Edad de los participantes (M $\pm \mathrm{DE})$ & $21,5 \pm 4,12$ \\
\hline \multicolumn{2}{|l|}{ Programa de estudios $(\%, n)$} \\
\hline Nutrición & $38,4 \%(38)$ \\
\hline Medicina & $19,1 \%(19)$ \\
\hline Odontología & $33,4 \%(33)$ \\
\hline Posgrado en área de la salud & $9,1 \%(9)$ \\
\hline \multicolumn{2}{|l|}{ Ocupación $(\%, n)$} \\
\hline Estudio & $67,6 \%(67)$ \\
\hline Estudio y trabajo & $32,4 \%(32)$ \\
\hline \multicolumn{2}{|c|}{$\begin{array}{l}\text { Tu plan de estudios, ¿requiere cursar de forma obligatoria alguna asignatura relacionada con los estudios } \\
\text { de género? }(\%, n)\end{array}$} \\
\hline Sí & $13,13 \%(13)$ \\
\hline No & $86,87 \%(86)$ \\
\hline \multicolumn{2}{|l|}{ ¿Existe en tu plan de estudios alguna asignatura optativa sobre género/igualdad de género? $(\%, n)$} \\
\hline si & $20,20 \%(20)$ \\
\hline no & $79,8 \%(79)$ \\
\hline \multicolumn{2}{|c|}{ Has cursado alguna asignatura sobre género/igualdad de género durante tu formación universitaria? (\%, $r$} \\
\hline Sí & 6,06\% (6) \\
\hline No & $93,94 \%(93)$ \\
\hline $\begin{array}{l}\text { Utilizando una escala de diez puntos siendo 1(mínima) y } 10 \text { (máxima), indica qué impor- } \\
\text { tancia otorgas a la formación para la igualdad de género ( } M \pm D E)\end{array}$ & $9,4 \pm 1,31$ \\
\hline
\end{tabular}


En la Tabla 2, se presentan los resultados del análisis de las subescalas de la ESFIG. En la subescala de sensibilidad institucional a la aplicación de la política de igualdad de género en los centros se encontró lo siguiente: respecto a si su facultad ha adoptado un enfoque proactivo hacia la igualdad de género la media de puntaje fue de 4,35 $\pm 1,03$. Al cuestionar sobre si el género recibe atención suficiente en las asignaturas se obtuvo una media de $3,78 \pm 1,35$. Por otro lado, la media obtenida sobre si su facultad aplica la normativa sobre igualdad fue de 4,47 $\pm 0,97$. En cuanto a si el plan desarrolla la competencia para educar en igualdad de género la media fue de 3,40 \pm 1.44 . Por último, respecto a si el profesorado está suficientemente sensibilizado la media fue de 3,73 \pm 1.36 .

En cuanto a la subescala sobre incorporación de la perspectiva de género en los planes de estudio se observó una media de 4,92 \pm 0,77. Específicamente sobre género integrado en la docencia con carácter obligatorio la media fue de 4,61 $\pm 1,15$. Sobre si existe al menos una asignatura sobre igualdad en el plan la media fue de 4,69 $\pm 1,18$. Para mayor atención a la diversidad de identidades sexuales y sobre si todas las asignaturas impartidas con perspectiva de género la media fue de 4,73 \pm 1,18 y 4,35 \pm 1,29 respectivamente. En el aspecto sobre perspectiva de género esencial para combatir el sexismo la media fue de 5,46 \pm 0,73 y para género igual de importante que otras diferencias la media fue de 5,20 \pm 0,83. Por último, en esta subescala la media para formación condición necesaria para educar en igualdad de género fue de 5,41 $\pm 0,84$.

En la subescala de desigualdades vinculadas al género en los procesos instructivos se encontró una media de 3,03 $\pm 1,23$. Sobre si los logros de las estudiantes se minimizan la media fue de $2,90 \pm 1,46$ y si los estudiantes reciben más atención que las estudiantes fue de 2,62 $\pm 1,32$. Finalmente, en cuanto a las expectativas más altas de las alumnas que de los alumnos y sobre los logros de las estudiantes se atribuyen al esfuerzo el promedio fue de $2,96 \pm 1,51$ y $3,66 \pm 1,61$ respectivamente.

\begin{tabular}{|c|c|}
\hline \multicolumn{2}{|l|}{ Género (ESFIG) } \\
\hline Subescalas & $\begin{array}{l}(n=99) \\
M \pm D E\end{array}$ \\
\hline Sensibilidad institucional a la aplicación de la política de igualdad de género en los centros & $4,35 \pm 1,03$ \\
\hline 1. Mi facultad ha adoptado un enfoque proactivo hacia la igualdad de género & $4,35 \pm 1,03$ \\
\hline 8. El género recibe atención suficiente en las asignaturas & $3,78 \pm 1,35$ \\
\hline 2. Mi facultad aplica la normativa sobre igualdad & $4,47 \pm 0,97$ \\
\hline 7. El plan desarrolla la competencia para educar en igualdad de género & $3,40 \pm 1,44$ \\
\hline 12. El profesorado está suficientemente sensibilizado & $3,73 \pm 1,36$ \\
\hline Incorporación de la perspectiva de género en los planes de estudio & $4,92 \pm 0,77$ \\
\hline 9. Género integrado en la docencia con carácter obligatorio & $4,61 \pm 1,15$ \\
\hline 11. Al menos una asignatura sobre igualdad en el plan & $4,69 \pm 1,18$ \\
\hline
\end{tabular}

M: Media, DE: Desviación Estándar. 


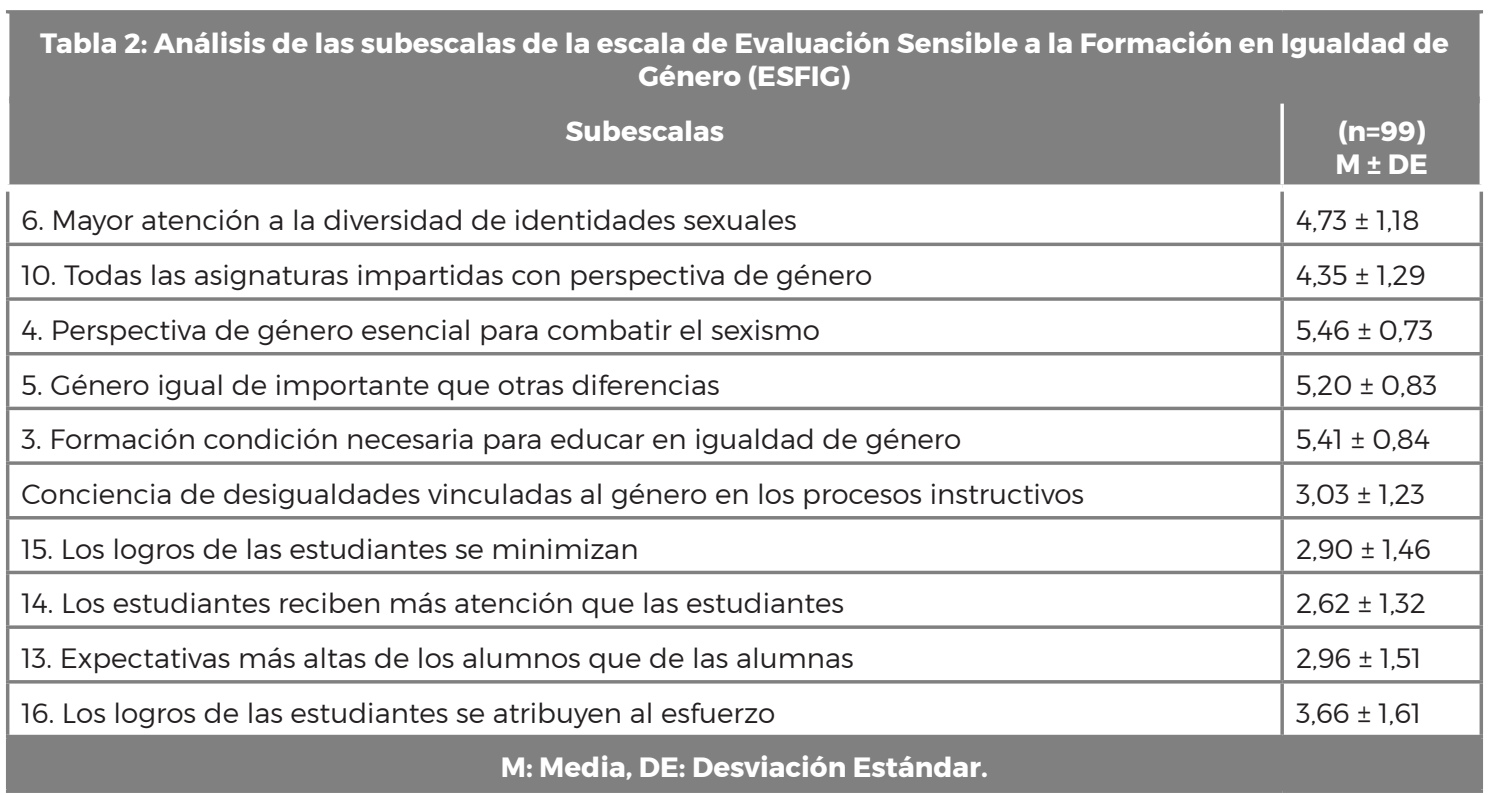

En la Tabla 3 se presenta la comparación sobre percepción de la aplicación de la perspectiva de género según el sexo asignado al nacer y el programa educativo al que se encuentran inscritos los estudiantes. Se observa que en cuanto a la sensibilidad institucional a la aplicación de la política de igualdad de género en los centros, la media para masculino fue de 4,00 \pm 0,99 y 3,92 \pm 0,88 para femenino, no se observaron diferencias estadísticamente significativas en cuanto al sexo $(p=0,176)$. Se encontró una diferencia significativa en cuanto al programa educativo, entre la percepción de los alumnos de Odontología $(4,03 \pm 1,04)$ con respecto a los de Medicina (3,95 $\pm 0,79)$, sin encontrar diferencias con respecto a los estudiantes de Nutrición $(4,05 \pm 0,82)$ y Posgrado en el área de la salud $(3,15 \pm 0,77$. No se observaron diferencias en la subescala de incorporación de la perspectiva de género en los planes de estudio en cuanto al sexo (4,71 \pm 1,02 masculino vs 5,00 \pm 0,63 femenino, $p=0,405)$. Al analizar si existían diferencias en cuanto al programa educativo en esta misma subescala no se observaron diferencias significativas $(p=0,470)$ siendo la media de 5,04 \pm 0,71 para nutrición, 4,80 \pm 0,79 para odontología, 4,79 \pm 0,64 para medicina y 5,09 \pm 1,17 para posgrado en ciencias en área de la salud.

Finalmente, en la comparación en la subescala sobre conciencia de desigualdades vinculadas al género en los procesos instructivos no se observaron diferencias en el sexo (2,74 $\pm 1,25$ y $2,74 \pm 1,25$ para masculino y femenino respectivamente, $\mathrm{p}=0,284$ ). Así mismo, al analizar las diferencias en cuanto al programa educativo en esta misma subescala se encontró un valor $\mathrm{p}$ de 0,107 considerado no estadísticamente significativo. Las medias obtenidas para el programa educativo en esta subescala fueron de: $3,05 \pm 1,14$ en nutrición, 2,86 \pm 1,30 en odontología, 3,25 \pm 1,25 en medicina y 3,11 \pm 1,43 en posgrado en ciencias en área de la salud. 
Tabla 3: Percepción de la aplicación de la perspectiva de género según el sexo asignado al nacer y el programa educativo al que se encuentran inscritos los estudiantes

\begin{tabular}{|c|c|c|}
\hline Subescalas & $\begin{array}{l}(n=99) \\
M \pm D E\end{array}$ & Valor de p \\
\hline \multicolumn{3}{|c|}{$\begin{array}{l}\text { Sensibilidad institucional a la aplicación de la política de igualdad de género } \\
\text { en los centros }\end{array}$} \\
\hline Sexo & & 0,176 \\
\hline Masculino & $4,00 \pm 0,99$ & \\
\hline Femenino & $3,92 \pm 0,88$ & \\
\hline Programa educativo & & $0,0315^{*}$ \\
\hline Nutrición & $4,05 \pm 0,82 a b$ & \\
\hline Odontología & $4,03 \pm 1,04 \mathrm{~b}$ & \\
\hline Medicina & $3,95 \pm 0,79 a$ & \\
\hline Posgrado en área de la salud & $3,15 \pm 0,77 \mathrm{ab}$ & \\
\hline \multicolumn{3}{|c|}{ Incorporación de la perspectiva de género en los planes de estudio } \\
\hline Sexo & & 0,405 \\
\hline Masculino & $4,71 \pm 1,02$ & \\
\hline Femenino & $5,00 \pm 0,63$ & \\
\hline Programa educativo & & 0,470 \\
\hline Nutrición & $5,04 \pm 0,71$ & \\
\hline Odontología & $4,80 \pm 0,79$ & \\
\hline Medicina & $4,79 \pm 0,64$ & \\
\hline Posgrado en área de la salud & $5,09 \pm 1,17$ & \\
\hline \multicolumn{3}{|c|}{ Conciencia de desigualdades vinculadas al género en los procesos instructivos } \\
\hline Sexo & & 0,284 \\
\hline Masculino & $2,74 \pm 1,25$ & \\
\hline Femenino & $3,15 \pm 1,21$ & \\
\hline Programa educativo & & 0,107 \\
\hline Nutrición & $3,05 \pm 1,14$ & \\
\hline Odontología & $2,86 \pm 1,30$ & \\
\hline Medicina & $3,25 \pm 1,25$ & \\
\hline Posgrado en área de la salud & $3,11 \pm 1,43$ & \\
\hline
\end{tabular}

M: Media, DE: Desviación Estándar. Prueba de T para muestras independientes, ANOVA y Prueba de Tukey, $\mathbf{p}<\mathbf{0 , 0 5}$. Literales diferentes indican diferencias significativas. 


\section{DISCUSIÓN}

En los resultados de esta investigación se encontró que, según lo que refieren los estudiantes universitarios, la mayoría de los programas educativos no requieren cursar una asignatura relacionada con igualdad de género. En esta población, la gran mayoría de los universitarios consideran importante la formación para igualdad de género, sin embargo, solamente un $6 \%$ ha cursado una asignatura relacionada con la perspectiva de género. Con el paso de los años se ha visto que cada vez más personas están interesadas en los temas de igualdad de género, en este caso de los ámbitos académicos, quizá haga falta dar a conocer a los universitarios las opciones disponibles de asignaturas optativas relacionadas a esta temática y generar más opciones que puedan cursar y/o participar en comités que lleven a cabo actividades con el objetivo de lograr la igualdad entre hombres y mujeres. Se requiere que las universidades de todos los países empiecen a implementar la perspectiva de género, la igualdad y la no discriminación para lograr cambios positivos en sus estudiantes y empleados y lograr con ello una sociedad más inclusiva y respetuosa (Donoso-Vázquez \& Velasco-Martínez, 2013).

En la mayoría de los programas educativos, son muy pocos los créditos que se dedican a las asignaturas en perspectivas de género, incluso en algunos solamente se abordan de manera aislada todos los temas relacionados con la igualdad de género (Merma-Molina, et al., 2017). En esta investigación los estudiantes perciben que sus planes de estudio no desarrollan de manera importante la competencia para educar a los universitarios en igualdad de género. Los estudiantes consideran que sus docentes no están suficientemente sensibilizados con la perspectiva de género, este aspecto es importante porque entonces pone de manifiesto que no se están promoviendo en el aula de clases las pautas para lograr igualdad entre hombres y mujeres y esto se confirma también cuando hacen referencia a que en las asignaturas no se está prestando atención suficiente al tema de género. Vale la pena resaltar que, en percepción de los estudiantes, la facultad si ha adoptado un enfoque proactivo hacia la igualdad de género y aplica la normativa sobre igualdad, pero falta que a través de los docentes en las distintas asignaturas se promueva una verdadera equidad entre ambos sexos.

Abordar los temas de perspectiva de género en las instituciones de educación superior resulta un tema incómodo y muchas veces difícil de tratar, sin embargo, se ha hecho mucho trabajo importante. Como ya se ha comentado en este trabajo, en las universidades existen comités o unidades conformadas por personas con experiencia en perspectiva de género para fomentar con el personal académico, administrativo y de servicio la igualdad entre hombres y mujeres. Sin embargo, sigue faltando la transversalidad de la perspectiva de género en los currículos (Ordorika, 2015). Los estudiantes que participaron en esta investigación, perciben que dentro de sus facultades se ha trabajado para combatir el sexismo y que se brindan siempre que es posible las condiciones necesarias para educar en igualdad de género. Sin embargo, consideran que falta incorporar la perspectiva de género en los planes de estudio e incluir temas de género en la capacitación que se brinda a los docentes. Por último, refieren que faltan asignaturas sobre igualdad en sus mapas curriculares, a pesar de que como parte de un programa institucional se cuenta con al menos una asignatura optativa sobre equidad de géneros.

En los tiempos que la sociedad vive actualmente, se da prioridad a la formación integral 
y multidisciplinaria, lo que es una oportunidad que se debe aprovechar para incluir la perspectiva de género en todas y cada una de las actividades de las Instituciones de Educación Superior. La lucha contra la discriminación solamente se puede lograr si todos los actores involucrados asumen un rol para educar a la población en temas de equidad, respeto y tolerancia (Alegre-Henderson, 2018). En los últimos años la perspectiva de género en la educación médica ha tomado un papel importante, por lo que desde los primeros semestres se sensibiliza en algunas universidades la importancia de la igualdad de género en la atención médica. Lo anterior permite una atención médica con perspectiva de género que esté libre de discriminación (Valenzuela \& Cartes, 2019).

Se ha visto que las mujeres docentes a diferencia de los docentes hombres son más propensos a apoyar a todos aquellos estudiantes que tienen alguna condición especial, además, que llevan más a la práctica todos los valores que se les enseña en casa y en el entorno educativo. Se requiere que, desde los primeros años de estudio, en ambos sexos se fomente el deseo de apoyar y respetar a los semejantes, con la finalidad de que sin importar el papel que cada uno desempeñe en la sociedad, sea siempre respetuoso e inclusivo con todas las personas sin importar su condición educativa, social o económica (Novo-Corti, et al., 2014). Basta de estigmatizar los roles de cada género, tanto hombres como mujeres deben tener la misma capacidad para tratar a los otros con respeto, amabilidad y empatía. Este trabajo se puede lograr con el apoyo de los padres en casa, pero también con el trabajo del docente en las aulas de clases.

En los resultados de esta investigación se encontró que algunos estudiantes perciben que no existe igualdad de condiciones entre hombres y mujeres en las reglas impuestas por los docentes en las aulas de clases, así como que se valora más el esfuerzo de los hombres en comparación con el de las mujeres. Las desigualdades en las aulas de clases se pueden erradicar solamente cuando se dejan de lado las ideas que socialmente se han adoptado respecto al papel que el hombre y la mujer debe desempeñar en la sociedad (Jara, 2021).

Los expertos coinciden en que para poder lograr una igualdad de género en la formación educativa se requiere de un nuevo modelo educativo. Este modelo debe partir de la idea que los centros educativos son espacios no solamente para lograr el proceso de enseñanzaaprendizaje, aquí también los estudiantes desarrollan distintas habilidades para relacionarse con sus compañeros, pero también para aprender nuevas actitudes y aprender a convivir en un ambiente de equidad. Dicho modelo debe partir de las diferencias entre hombres y mujeres y dar prioridad a todos aquellos grupos que han sido discriminados a la vez que resalte los aportes de las mujeres en los distintos campos del saber (Durán, 2022).

En los programas educativos de medicina, suele darse al hombre un papel de proveedor de su familia, capaz de revertir en cualquier momento su situación para tener un mejor panorama y una persona que puede soportar el dolor con más facilidad que la mujer. Por su parte, se sitúa a la mujer como aquella persona que tiene un rol de cuidar a todos los que están a su alrededor, como un ser humano que es muy vulnerable a enfermedades tanto física como mentales. Todo esto pone en una clara desventaja a la mujer en comparación con el hombre y requiere ser abordado para erradicar las desigualdades que todo ello pueda ocasionar (Soledad-Salas, 2018). Pese a que los estudiantes de medicina están a cargo de la salud general de la población, muchos de ellos no saben qué organismos pueden atender a mujeres víctimas de violencia de género, así como de los protocolos que estas instituciones siguen para poder ayudar a las víctimas. Cada vez los futuros médicos tienen 
más conocimiento sobre todo lo que implica la violencia de género gracias al esfuerzo que hacen las instituciones educativas, así como de toda la información que llega a través de distintos sitios de internet, sin embargo, desconocen cómo actuar en cada uno de los casos, es decir, a quién acudir y cómo solicitar apoyo (Matorras, et al., 2021).

En un estudio realizado en una universidad de Argentina, se encontró que en la facultad de medicina cada vez existen más mujeres desempeñando el papel de docentes, pero sin ocupar a diferencia de los hombres, puestos de mayor jerarquía que poseen un mayor reconocimiento social y salarial. A diferencia de los hombres, las mujeres tienen solamente puestos interinos y ocupan puestos que tienen una mayor demanda que los de los hombres (Marconi, et al., 2020). Esto indica que falta mucho por recorrer para lograr una verdadera igualdad entre hombres y mujeres en el ámbito educativo y laboral. Fuera del contexto educativo, las médicas que se desempeñan en hospitales, se enfrentan a un mundo en el que el hombre es privilegiado con los mejores puestos, horarios, sueldos y un mayor reconocimiento (Manrique, et al., 2019).

\section{CONCLUSIÓN}

Las facultades en las que los universitarios que participaron en este proyecto, se encuentran trabajando arduamente en perspectiva de género y dicho esfuerzo es percibido y valorado por los universitarios. En general, hace falta que las universidades incluyan en sus programas de estudios más asignaturas relacionadas con la perspectiva de género, que se promuevan con los estudiantes y que sus docentes reciban una mayor capacitación en este tema. Lo anterior permitirá que en los espacios educativos se fomente en los futuros profesionistas el respeto, equidad y justicia, que traerá consigo mejores ciudadanos y una sociedad más inclusiva. Se ha hecho mucho trabajo en las distintas universidades para promover la igualdad de género, pero faltan aspectos claves importantes de lograr en las aulas de clases para lograr que las mujeres tengan las mismas oportunidades que los hombres en los espacios educativos, laborales y sociales.

\section{REFERENCIAS}

Alegre Henderson, M. (2018). La incorporación de la perspectiva de género en los planes de estudio de la PUCP. Revista Entorno. 66, pp. 10-17 http://hdl.handle.net/11298/869

Aragón Macías, L., Arras Vota, A. M. D. G., \& Guzmán Ibarra, I. (2020). Realidad actual de la elección de carrera profesional desde la perspectiva de género. Revista de la Educación Superior, 49(195), pp. 35-54. http://resu.anuies.mx/ojs/index.php/resu/article/view/1250

Manrique F de M., Amaya-Castellanos C.I, Rivero-Rubio C. (2019). Gender inequalities in the health setting: The case of medicine. Aquichan 19(2): e1927. DOI: https://doi.org/10.5294/ aqui.2019.19.2.7

Camarena Adame, M. E., \& Saavedra García, M. L. (2018). La perspectiva de género en los programas de estudio de las licenciaturas contables administrativas. Nóesis. Revista de 
ciencias sociales y humanidades, 27(54), 39-58. https://doi.org/10.20983/noesis.2018.2.3

Canosa Domínguez, N. M., Groba, B., Nieto-Riveiro, L., \& Pousada, T. (2020). Perspectiva de género en Ciencias de la Salud: Igualdad y Terapia Ocupacional. In IV Xornadas de Innovación Docente. pp. 295-304. Universidade da Coruña, Cufie. http://hdl.handle. net/2183/26018

Castillo, R. A. M., \& Reyes, C. M. (2022). Una retrospectiva de la desigualdad de género en la educación superior en el Estado de Hidalgo, México 2010. Acción y Reflexión Educativa, (47), pp. 43-60. https://www.revistas.up.ac.pa/index.php/accion_reflexion_educativa/ article/view/25822

Cerna, D. C. (2017). Desafíos para la institucionalización de la perspectiva de género en instituciones deeducaciónsuperioren México. Unamiradaaloscontextosorganizacionales. Revista Punto Género, (8), pp. 20-38. https://doi.org/10.20983/noesis.2018.2.3

Donoso-Vázquez, T., \& Velasco-Martínez, A. (2013). ¿Por qué una propuesta de formación en perspectiva de género en el ámbito universitario? Profesorado. Revista de currículum y formación de profesorado, 17(1), pp. 71-88.

Durán, M. P. (2022). Currículo oculto y no tan oculto de género en la educación superior. Revista Reflexiones, 101(2). DOI: https://doi.org/10.15517/rr.v101i2.45869

González Baltazar, R., Hidalgo Santacruz, G., Cortés, S., Contreras Estrada, M. I., Aldrete Rodríguez, M. G., Hidalgo González, B. J., \& Barrera Vega, J. A. (2015). Relación entre género y calidad de vida laboral en profesionales de salud. Psicogente, 18(33), pp. 52-65. https:// www.redalyc.org/pdf/4975/497551992006.pdf

Ingaramo, M. A. (2013). Los desafíos de la perspectiva de género en la definición de la agenda gubernamental. Revista Cátedra Paralela, (10), pp. 105-120. https://doi.org/10.35305/ cp.vi10.235

Jara, M. (2021). Discriminación, desigualdad y género. Participación de los varones y las mujeres en las aulas del Instituto de Profesores Artigas durante el año 2021. http:// repositorio.cfe.edu.uy/handle/123456789/1592

Jiménez Quenguan, M., \& Galeano Barbosa, D.J. (2020). La necesidad de educar en perspectiva de género. Revista Educación, 44(1), pp. 490-508. https://dx.doi.org/10.15517/revedu. v44i1.38529

Jiménez, R. C. (2015). Aspectos relevantes para la transversalización de la perspectiva de género en el proceso de desarrollo curricular universitario. Revista Espiga, (29), pp. 33-43. https://doi.org/10.22458/re.v14i29.959

Marconi, Agustina, Knopoff, Edgardo Gabriel, Irurzun, María Alejandra, Rojo, Carlos, Negri, Claudia Viviana, Freddi, Ivanna J., \& Chiarelli, Julieta. (2020). Docencia universitaria en salud: análisis de la distribución entre tipos de cargo y género. Salud(i)Ciencia, 23(8), pp. 619625. Epub 10 de abril de 2020.https://dx.doi.org/www.dx.doi.org/10.21840/siic/161236

Matorras, L., Servín R. y Auchter, M. (2021). Los estudiantes de medicina frente a la violencia de género año 2020. Libros de artículos científicos en salud. https://med.unne.edu.ar/ wp-content/uploads/2021/10/2021_23.pdf

Merma-Molina, G., Ávalos Ramos, M. A., \& Martínez Ruiz, M. Á. (2017). La igualdad de género en la docencia universitaria. Percepciones del alumnado. La manzana de la discordia. 12(1), pp. 103-115. doi: https://doi.org/10.25100/lamanzanadeladiscordia.v12i1.5552

Miralles-Cardona, Cristina, \& Cardona-Moltó, María Cristina, \& Chiner, Esther (2020). La perspectiva de género en la formación inicial docente: estudio descriptivo de las percepciones del alumnado. Educación XX1, 23(2), pp. 231-257. [fecha de Consulta 13 
de enero de 2022]. Recuperado de: https://www.redalyc.org/articulo.oa?id=70664431010 Moctezuma Navarro, David, Narro Robles, José, \& Orozco Hernández, Lourdes. (2014). La mujer en México: inequidad, pobreza y violencia. Revista mexicana de ciencias políticas y sociales, 59(220), pp. 117-146. Recuperado de http://www.scielo.org.mx/scielo. php?script=sci_arttext\&pid=S0185-19182014000100005

Novo-Corti, I., Muñoz-Cantero, J.-M., \& Calvo-Babio, N. (2014). Los futuros docentes y su actitud hacia la inclusión de personas con discapacidad. Una perspectiva de género. Anales de Psicología / Annals of Psychology, 31(1), pp. 155-171. https://doi.org/10.6018/ analesps.31.1.163631

Ordorika, I. (2015). Equidad de género en la Educación Superior. Revista de la educación superior, 44(174), pp. 7-17. http://www.scielo.org.mx/scielo.php?script=sci_ arttext\&pid=S0185-27602015000200001\&lng=es\&tlng=es

Perales, R. G. (2012). La educación desde la perspectiva de género. Ensayos: Revista de la Facultad de Educación de Albacete, (27), pp.1-18. https://doi.org/10.18239/ensayos. v27i0.81

Rodríguez, J. L. E., Ramírez, A. M., \& Vidaña, B. G. (2016). Perspectiva de género en México: Análisis de los obstáculos y limitaciones. Opción: Revista de Ciencias Humanas y Sociales, (13), pp. 12-36. https://www.redalyc.org/pdf/310/31048483002.pdf

Soledad Salas, M. (2018). Estereotipos de género ocultos en el aprendizaje de los estudiantes de Medicina: un análisis necesario. Edumecentro, 10(4), pp. 20-36.

Valenzuela V. Amanda, Cartes V. Ricardo. (2019). Perspectiva de género en la educación médica: Incorporación, intervenciones y desafíos por supercar. Rev. chil. obstet. ginecol. 84( 1 ): 82-88. Disponible en: http://dx.doi.org/10.4067/S0717-752620190001000822 